\title{
Correction to: Contemporary Russia
}

\section{Edwin Bacon}

This book was published with incorrect imprint and published information. This has now been corrected.

The updated online version of the original book can be found at https://doi.org/10.1007/978-1-137-40827-3

(C) Edwin Bacon, 2021

E. Bacon, Contemporary Russia, DOI 10.1007/978-1-137-40827-3_11 